\title{
Emerging chemical heterogeneities in a commercial 18650 Li-ion battery during early cycling
}

Matras D. ${ }^{a, b, *}$, Ashton T. E. ${ }^{c}$, Dong H.c, Mirolo M. ${ }^{d}$, Martens I. ${ }^{d}$, Drnec J. ${ }^{d}$, Darr J.A. ${ }^{c}$, Quinn P. D. ${ }^{b}$, Jacques S.D.M. ${ }^{e}$, Beale A. M..$^{\mathrm{ce}, \mathrm{f}}$, Vamvakeros A. ${ }^{\mathrm{e}, *}$

a) The Faraday Institution, Quad One, Harwell Science and Innovation Campus, Didcot, OX11 ORA, United Kingdom

b) Diamond Light Source, Harwell Science and Innovation Campus, Didcot, Oxfordshire OX11 ODE, United Kingdom

c) Department of Chemistry, University College London, 20 Gordon Street, London WC1H 0AJ, United Kingdom

d) ESRF - The European Synchrotron, Grenoble 38000, France

e) Finden Limited, Merchant House, 5 East St Helens Street, Abingdon, OX14 5EG, United Kingdom

f) Research Complex at Harwell, Rutherford Appleton Laboratory, Harwell Science and Innovation Campus, Didcot, Oxon OX11 OFA, United Kingdom

*correspondence email: matras.dorota@gmail.com, antony@finden.co.uk

\section{Abstract}

X-ray diffraction computed tomography (XRD-CT) was employed to study a commercial 18650 cylindrical $\mathrm{LiNi}_{0.8} \mathrm{Co}_{0.15} \mathrm{Al}_{0.05} \mathrm{O}_{2}$ (NCA) battery under operating conditions and during seven cycles. Multiple chemical heterogeneities related to the lithium distribution were observed in both the cathode and the anode from the analysis of the spatially-resolved diffraction signals. It is shown that during the battery charging, the anode exhibits different degrees of activity regarding the lithiation process. Explicitly, the following three regions were identified: uniform/homogenous 
lithiation, delayed lithiation and inactive-to-lithiation regions. The delayed lithiation region, observed for the first time, was seen to have a direct impact on the properties of NCA in its close proximity during the battery discharging, preventing its full lithiation by trapping the lithium in the form of $\mathrm{LiC}_{30-30+}$ phase. Further to this, the aluminum tab negatively affected the NCA in direct contact with it, leading to different lattice parameter a and c evolution compared to the rest of the cathode.

\section{Introduction}

To meet the continuous increase in global energy demand while imposing restrictions on greenhouse gas levels typically generated by fossil fuels, requires alternative energy storage technologies. It has been realised that Li-ion batteries ( $\mathrm{LiBs}$ ) have the potential to bridge this gap and reach energy sustainability. LiBs are considered the main power source for the next generation of electric vehicles (EVs) and the advancements achieved over the past decade in LiB technology, in terms of both battery materials chemistry and system management/engineering, have resulted in more affordable EV batteries with increased mileage per charge ${ }^{1-6}$. The energy density of EV batteries is primarily governed by the capacity of the cathode material; currently the cathode used in commercial batteries is based on layered $\mathrm{LiMO}_{2}$-type materials (where $\mathrm{M}=\mathrm{Co}$, $\mathrm{Ni}, \mathrm{Mn}$ and $\mathrm{Al}$ ) with theoretical capacities in the order of $200-250 \mathrm{mAh} \cdot \mathrm{g}^{-1}$. Growing ethical concerns related to the conditions at Co mining facilities coupled with its limited availability led to the development of layered cathode materials with significantly reduced amount of Co; Ni-rich cathode materials containing $80 \%$ of nickel, $\mathrm{LiNi}_{0.8} \mathrm{Co}_{0.15} \mathrm{Al}_{0.05} \mathrm{O}_{2}(\mathrm{NCA})$ and $\mathrm{LiNi}_{0.8} \mathrm{Co}_{0.1} \mathrm{Mn}_{0.1} \mathrm{O}_{2}$ (NMC811) have been recently successfully commercialised as cathode materials for the EV batteries. The substitution of toxic and expensive Co with Ni however comes at a price; Ni rich layered materials have reduced stability and exhibit a decrease in capacity over prolonged 
cycling, especially when operating at high temperatures and voltages (i.e. above $\sim 4.2 \mathrm{~V}$ ). Various mechanisms have been identified as responsible for the degradation in these materials, including both reactions and phase transformations occurring at the surface and within the bulk, leading to dissolution and cracking of active materials and formation of phases that suppress the $\mathrm{Li}$ movement within the electrode ${ }^{7-11}$.

There is a wealth of knowledge related to degradation at the device level, most commonly characterised through cycling stability, but the phenomena that can contribute to capacity fade and/or cell failure are difficult to decouple experimentally due to their often multi-length scale nature (i.e. scale and resolution are almost always linked). For instance, cylindrical cells are known to exhibit heterogeneities in temperature and state of charge which can have a negative impact on the overall cell performance over prolonged periods of cycling ${ }^{12-16}$. In addition, the manufacturing process of the various cell components can significantly affect the state of the active materials ${ }^{17}$, introducing defects (e.g. particle cracking during calendering and active material layer delamination) responsible for reduced electrochemical capacity ${ }^{18,19}$. Obtaining a chemical insight from within a real working device is therefore essential to achieve a thorough understanding of the evolving chemistry and possible aging mechanisms. Typically, battery materials are extracted from the device(s) and characterised by ex situ spectroscopy, scattering and microscopy-based techniques; the results are then compared against the initial state of active materials and subsequently the changes observed in the electrochemical performance data collected during the device operation (i.e. differential voltage analysis or electrochemical impedance measurements) are correlated to changes in the battery materials ${ }^{20-22}$. Large cells can be also studied in a non-destructive way using high energy $\mathrm{X}$-rays and neutrons; techniques based on scattering and absorption have been proven to provide invaluable insight into the evolving solid-state chemistry and morphological/structural changes of a cycled battery (both in situ and ex situ) ${ }^{23-36}$. More specifically, combining imaging/tomography with X-ray 
diffraction/scattering signal (e.g. X-ray diffraction computed tomography aka. XRD-CT) or neutron scattering (i.e. direct neutron imaging) allows for capturing the various chemical heterogeneities present across the battery cell which can be directly related to current heterogeneities ${ }^{37-40}$. The order of magnitude higher spatial resolution achieved with XRD-CT images when compared to

neutron diffraction imaging (e.g. $0.02 \times 0.02 \times 0.003 \mathrm{~mm}$ vs $2 \times 2 \times 20 \mathrm{~mm}$ respectively ${ }^{37,39,40}$ ) enables the visualisation of gradients in the distribution of lithiated graphite phases within one electrode as a function of cycling rate.

In this work, we present results from an operando X-ray diffraction computed tomography (XRDCT) experiment with a commercial 18650 NCA battery. Diffraction point measurements were collected during early cycling stages to investigate the evolving solid-state chemistry within the battery cell. In addition, multiple XRD-CT datasets were acquired at different potentials while charging the cell in order to visualise the state-of-charge heterogeneities. These are observed through the distribution of lithiated graphitic phases as well as the average lattice parameters of cathode layered oxide. Data analysis was performed with a deep learning approach using the state-of-the-art Parameter Quantification Network (PQ-Net) which allowed us to extract crystallographic information, from both point measurements and XRD-CT data, orders of magnitude faster compared to conventional approaches (ca. $3 \mathrm{~min}$ vs $10 \mathrm{hr}$ for analysis of one XRD-CT dataset with the PQ-Net and conventional full profile analysis using the Rietveld method with the TOPAS software respectively) ${ }^{41}$.

\section{Results and discussion}

The contrast in XRD-CT images arises from differences in signal collected from X-rays scattered by long-range ordered components present in the sample. This allows the identification of crystalline and semicrystalline components and visualisation of their distribution in the 
reconstructed cross sections. Figure 1 presents the distribution of all identified components in the cross-section taken at the middle of the cell commercial cylindrical 18650 battery cell charged to 4.2 V (see Methods section for details regarding the cycling protocol); the cell consisted of stainless steel casing (Fe), centre pin, semicrystalline separator, anode tab, anode Cu current collector, graphite, four partially lithiated graphite phases $\mathrm{Li}_{x} \mathrm{C}$, cathode $\mathrm{Al}$ current collector and NCA phase $\left(\mathrm{Li}_{x} \mathrm{Ni}_{0.81} \mathrm{Co}_{0.11} \mathrm{Al}_{0.08} \mathrm{O}_{2}\right)$. The anode was found to be composed of four different crystalline phases: $\mathrm{LiC}_{6}, \mathrm{LiC}_{12}, \mathrm{LiC}_{30}$ and $\mathrm{LiC}_{30+}$ with the major phase, $\mathrm{LiC}_{12}$, uniformly distributed in the anode and $\mathrm{LiC}_{6}$ present primarily at the electrode surface (Figure $1 \mathrm{~b}$ ). $\mathrm{LiC}_{30}, \mathrm{LiC}_{30+}$ and graphite were co-located in the regions adjacent to the Al tab current collector (Figure 1a), which is in agreement with our previous observations regarding the effect of battery cell components on chemical heterogeneities (i.e. regions of the Al current collector, steel casing and polymer separator in the core of the jelly roll) ${ }^{42}$. It is important to note that the identification of the various partially lithiated graphite phases was only possible through the analysis of spatially-resolved diffraction patterns; as we later show these signals would be difficult to identify when performing single point measurements. Regarding the $\mathrm{Li}_{x} \mathrm{C}$ phases, their structure is well known and described in previous studies ${ }^{27,43-46}$ apart from the low-lithiated graphite phase, referred in this study as $\mathrm{LiC}_{30+}$ with its principal reflection present at $d$-spacing of $\approx 3.40 \AA$ or $Q$ of $\approx 1.85 \AA^{-1}$ (in other studies this is also referred as $\mathrm{Li}_{1-x} \mathrm{C}_{54}{ }^{47}$ ). More information regarding this phase can be found in the Supporting Information (Table S1-S2). Regarding the composition of the centre pin and anode tab, diffraction patterns showed that both phases can be described as cubic with lattice parameters of $3.594 \AA$ and $3.529 \AA$ respectively (Table S1-S2); it is indicated that the anode tab consists of the metallic alloy $\mathrm{Ni}_{0.92} \mathrm{Cu}_{0.02}$ while the center pin is based on a Fe metallic alloy. These materials are known to be used in the manufacturing of cylindrical Li-ion batteries ${ }^{48}$. 
a
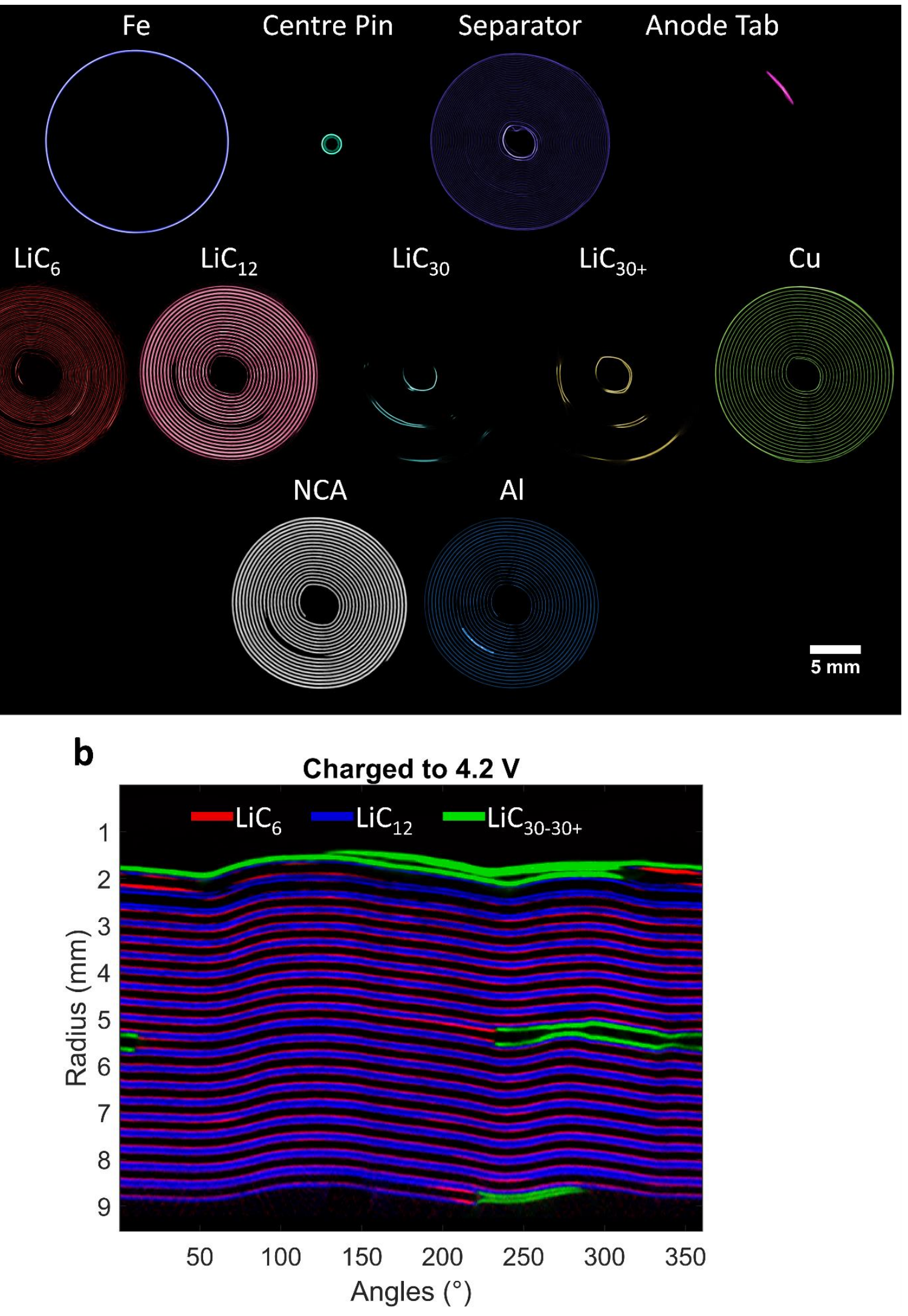
Figure 1. Spatial distribution of all components identified in the charged NCA battery cell. Panel a: First row presents the main cell components (Fe casing, centre pin, polymer separator, anode tab), the second row presents anode phases ( $\mathrm{Li}_{x} \mathrm{C}$ phases) and the anode current collector $(\mathrm{Cu})$ and the third row presents cathode phase and the cathode current collector (Al). Panel b: virtually unrolled battery electrode charged to $4.2 \mathrm{~V}$ showing the distribution of $\mathrm{LiC}_{6}, \mathrm{LiC}_{30-30+}$ and $\mathrm{LiC}_{12}$,

X-ray diffraction point measurements performed during the first two full cycles were used to follow the evolution of crystalline lithiated graphite phases (Figure 2). It is important to note that for the point measurements analysis only four phases were used to describe the anode composition: graphite, $\mathrm{LiC}_{30}, \mathrm{LiC}_{12}$ and $\mathrm{LiC}_{6}$ as the complexity of the model with additional $\mathrm{LiC}_{30+}$ and $\mathrm{LiC}_{18}$ (i.e. six phase model) led to unstable results. This is a common problem due to insufficient angular (2theta) resolution as well as multiple peak overlap in the region where the highest reflections of graphite and the various lithiated graphite phases are present ${ }^{49,50}$. The lattice parameters of the four phases were refined to fit the experimental data and the regions covered by these phases (in Q space) can be found in the Supporting Information (Table S3). However, as shown in Figure 1, the presence of the various $\mathrm{Li}_{x} \mathrm{C}$ phases can be resolved with the XRD-CT images.

In the fully discharged state (i.e. the as-received cell was first discharged to $2.65 \mathrm{~V}$ ), the anode consisted mainly of the graphite phase. After applying a current (3.5 A corresponding to 1C), leading to a subsequent increase in voltage, the quantity of graphite rapidly decreased resulting in the formation of the lithiated graphite phase $\mathrm{LiC}_{30-30+}$ with its highest quantity at $\sim 3.7 \mathrm{~V}$. Following this, the $\mathrm{LiC}_{30-30+}$ gradually decreased to $~ 25$ wt. \% while the $\mathrm{LiC}_{12}$ gradually increased to $\sim 55$ wt. \%. After reaching $4 \mathrm{~V}$ the quantity of $\mathrm{LiC}_{6}$ started to increase and reached $20 \mathrm{wt} \% \%$ at 4.2 V. At 4.2 V the applied current was removed and the cell was allowed to relax for a time period of $10 \mathrm{~min}$. An XRD-CT dataset was then collected and afterwards the cell was discharged to 2.65 $\mathrm{V}$ with $1 \mathrm{C}$ rate (applying $-3.5 \mathrm{~A}$ current). During the relaxation time and the $\mathrm{XRD}-\mathrm{CT}$ scan 
(relaxation periods are not included in Figure 2), the solid-state chemistry was slowly and gradually changing, resulting in a decrease of the $\mathrm{LiC}_{6}$ phase (from 20 wt. \% to 10 wt. \%) and an increase of the $\mathrm{LiC}_{12}$ phase (from $55 \mathrm{wt}$ \% to $70 \mathrm{wt}$ \%) (i.e. during the relaxation the lithium is re-equilibrating between the partially reduced phases). During the discharge, the quantity of $\mathrm{LiC}_{12}$ gradually decreased and completely disappeared at $\sim 3 \mathrm{~V}$. Simultaneously, the $\mathrm{LiC}_{30-30+}$ phase increased to 50 wt. $\% \sim 3.1 \mathrm{~V}$ and then decreased again to $\sim 20 \mathrm{wt}$ \% for the discharged cell. The graphite reappeared in the anode $\sim 3.2 \mathrm{~V}$ and reached $80 \mathrm{wt} . \%$ for the discharged cell. The major difference between charge and discharge is based on the lithiation mechanism; the charge can be described as a continuous phase evolution in the range between graphite and $\mathrm{LiC}_{12}$ whereas the discharge occurs via a simultaneous growth of two phases $\mathrm{Li}_{c 30-30+}$ and graphite with the latter one appearing below 3.3 V. The second cycle showed the same solid-state evolution regarding the amount of phases and the voltages at which the various phases appeared or disappeared. 

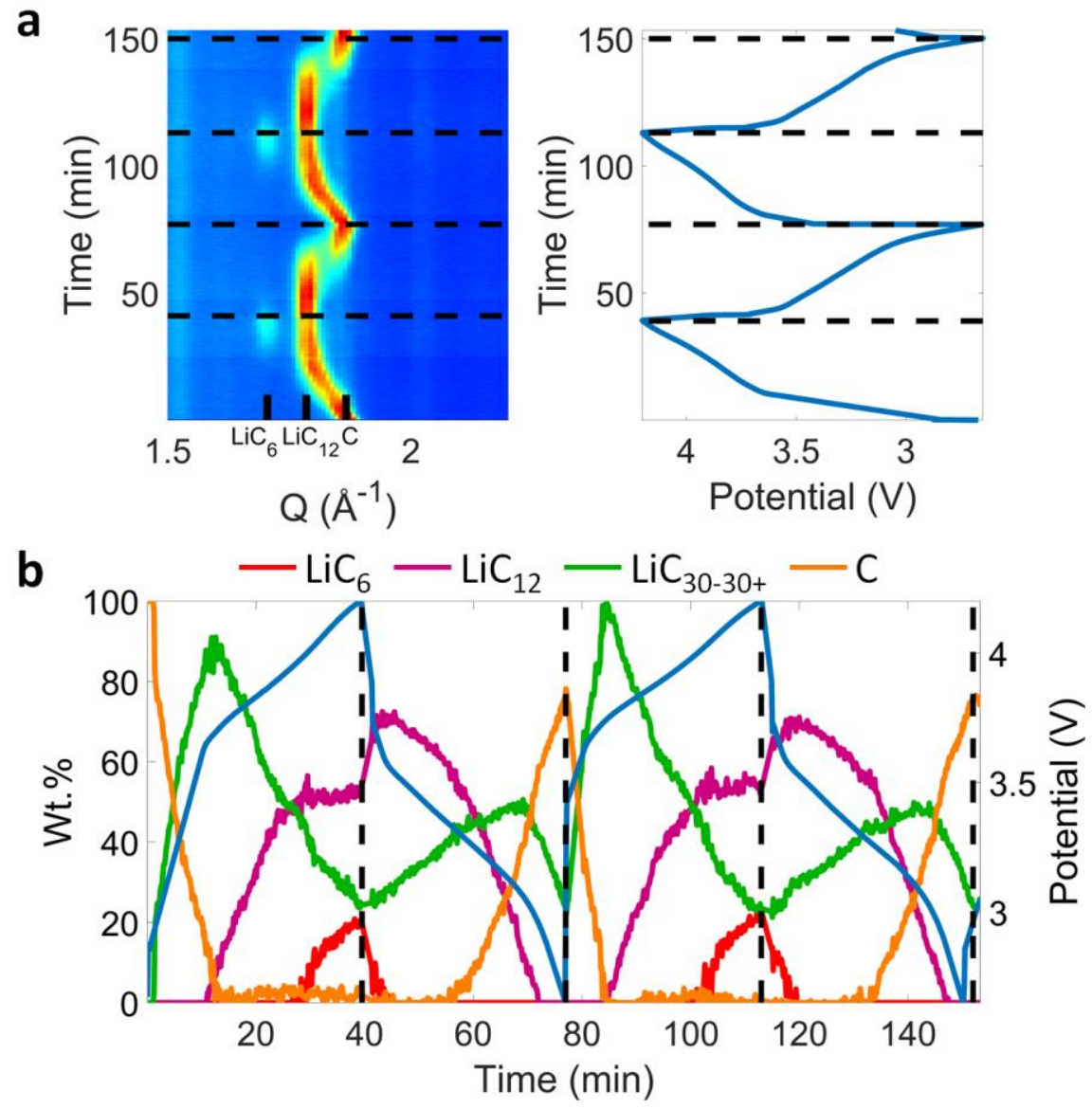

Figure 2. Solid-state chemistry evolution in the anode during the first two full cycles (charging-discharging performed with $1 \mathrm{C}$ rate). Panel a: XRD point measurements as a function of time and the corresponding voltage plot, Panel b: Weight percent evolution of lithiated graphite phases in the anode and the corresponding voltage plot (blue line). Note: the dashed line marks the time where the XRD-CT data were collected.

Figure 3 presents the distribution of the crystalline phases identified in the anode for the charged cell to $4.2 \mathrm{~V}$ and for the discharged cell to $2.65 \mathrm{~V}$ for two full cycles (more information regarding the phase identification can be found in the Supporting Information Figure S1-4, Table S4). At the charged state, the anode is composed primarily of $\mathrm{LiC}_{12}$, uniformly distributed in the electrode, as well as $\mathrm{LiC}_{6}$ and $\mathrm{LiC}_{30-30+}$ phases. The $\mathrm{LiC}_{6}$ was formed mainly at the anode surface (Figure 1b) while $\mathrm{LiC}_{30-30+}$ was found to be present in specific regions that can be identified as close to the $\mathrm{Al}$ 
tab and separator (Figure 3b). For the discharged state, the anode consisted of graphite, uniformly distributed in the cell, as well as the $\mathrm{LiC}_{30-30+}$ phases which appeared also in additional regions. These are present in the radial range between the $\mathrm{Al}$ tab and the steel casing; these regions are mentioned throughout the text as "middle-region" (indicated by the arrows in Figure 3). When comparing the distribution of $\mathrm{LiC}_{30-30+}$ between the charged and discharged state, it can be seen that there are certain regions where the lithiation and delithiation of the anode is suppressed; these regions are in close proximity to the Al tab current collector and separator. The Al tab current collector and separator were previously shown to influence the lithium distribution in both the anode and cathode of AAA Li-ion commercial batteries ${ }^{42}$. Our results indicate that a similar phenomenon can be observed in a cell of different size and battery cathode chemistry. It should be pointed out that no $\mathrm{LiC}_{18}$ was observed to form at the charged or discharged state. 

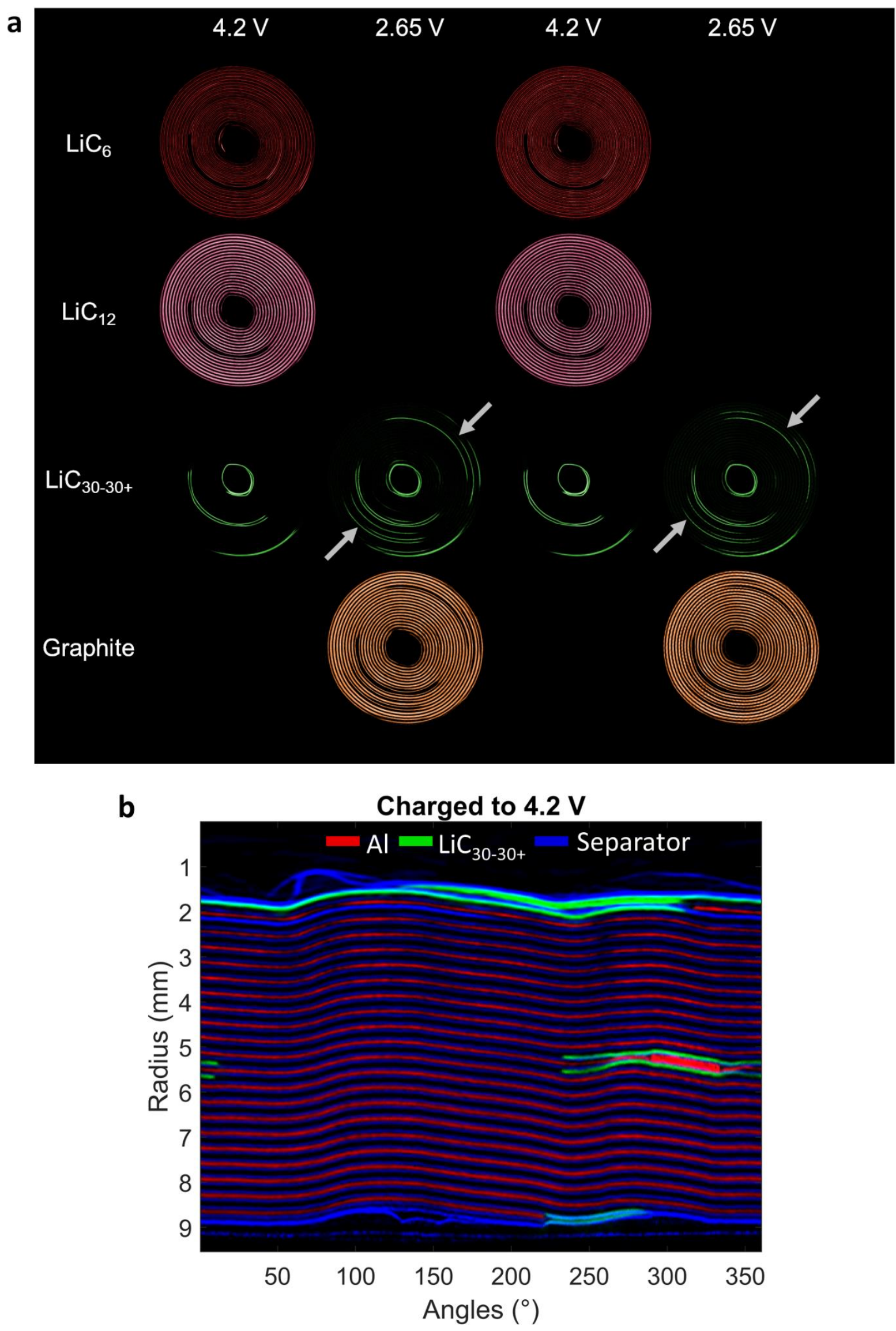
Figure 3. Distribution of anode phases identified in the charged to $4.2 \mathrm{~V}$ and discharged to $2.65 \mathrm{~V}$ battery cell during the first two cycles. Panel a: Spatial distribution obtained from the analysis of the XRD-CT data. Panel b: virtually unrolled battery electrode charged to $4.2 \mathrm{~V}$ showing the distribution of $\mathrm{Al}, \mathrm{LiC}_{30-30+}$ and separator.

Figure 4 presents the distribution of lattice parameters a and c of the NCA phase in the cell charged to $4.2 \mathrm{~V}$ and discharged to $2.65 \mathrm{~V}$. Despite the NCA phase being uniformly distributed in the cathode (Figure 1), differences in the distribution of lattice parameters could be identified in both the charged and discharged state. More precisely, in the region of the cathode which is directly connected to the Al tab, variations in the lattice parameters could be observed (Figure S5-S7, Table S5); for the charged state the lattice parameter a was observed to be higher than the average lattice parameter a for the rest of the cathode and inversely for the lattice parameter c (i.e. lattice parameters c lower). The difference between the values of lattice parameters c (and a) can be used as an indication of the lithium distribution in the cathode; higher lattice parameter c suggests that the cathode phase contains less lithium in its structure ${ }^{24,51,52}$. This comparison can be made by assuming that both materials belong to the same hexagonal cell. Regarding the discharged state, heterogeneities in lattice parameters can be observed again for the cathode materials connected to the Al tab, but additionally in other areas (NCA ROI1 in Figure 4b). To clarify, NCA ROI1 corresponds to cathode material directly connected to the Al tab and a few layers in the middle-region while NCA ROI2 corresponds to the rest of the electrode layers. Interestingly, the regions shown by $\mathrm{ROI} 1$ in Figure $4 \mathrm{~b}$ are in close proximity to anode regions where the $\mathrm{Li}_{30-30+}$ phases were also observed for the discharged state. These observations suggest that for the discharge state, the cathode lithiation did not occur uniformly. The aforementioned areas (i.e. in the middle of the cell) contain the NCA structure with lower lattice parameter a and higher lattice parameter $\mathrm{c}$, which suggests lower amounts of lithium in the cathode material. This is in agreement with the distribution of lithium in the anode, as these 
regions were seen to contain the $\mathrm{LiC}_{30-30+}$ phase instead of the fully de-lithiated graphite phase. In addition, the lattice parameters, and hence the amount of lithium, in the cathode material directly connected to the Al tab slightly changes between charged and discharged state. It should be noted that all the changes in the NCA lattice parameters (i.e. expansion vs contraction) are reversible and no difference between the various cycles are observed.

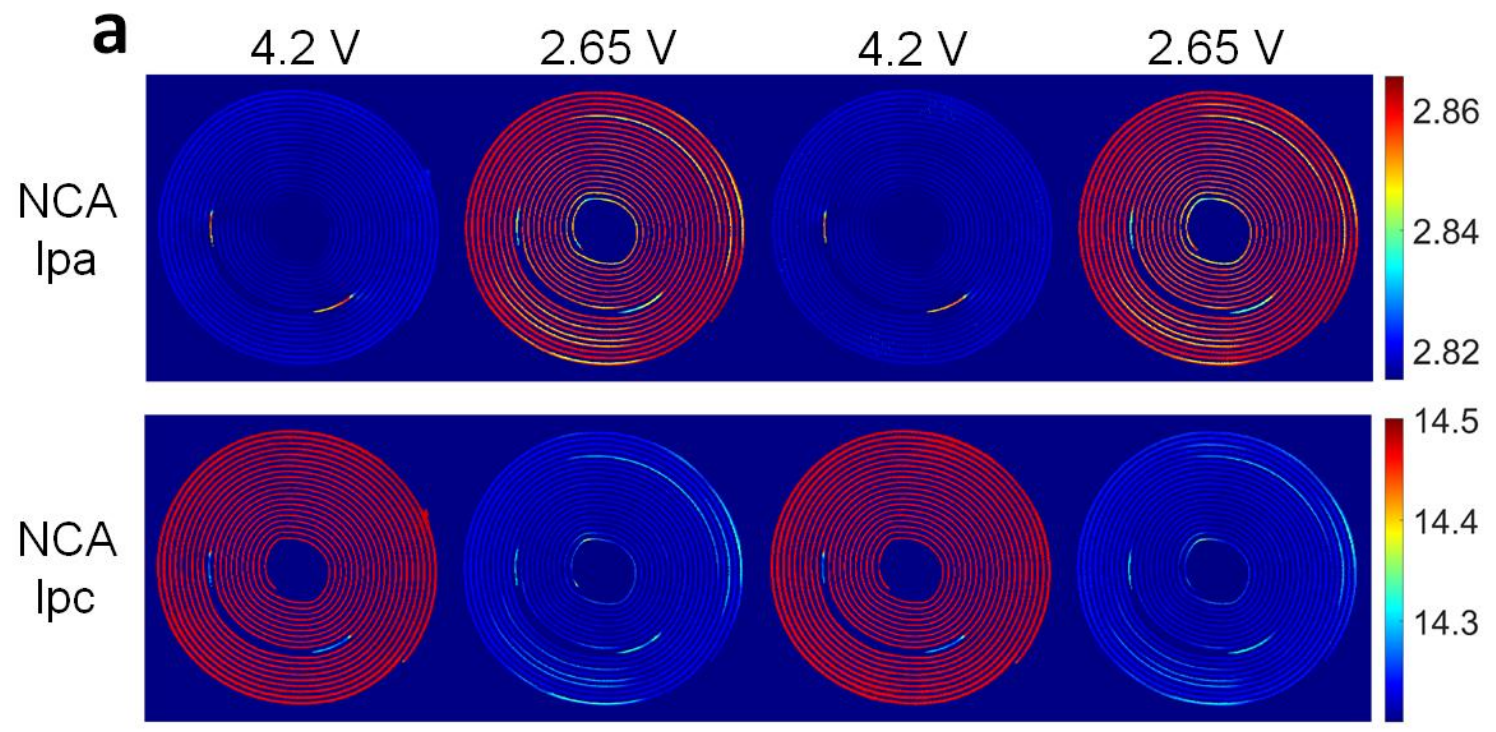

b

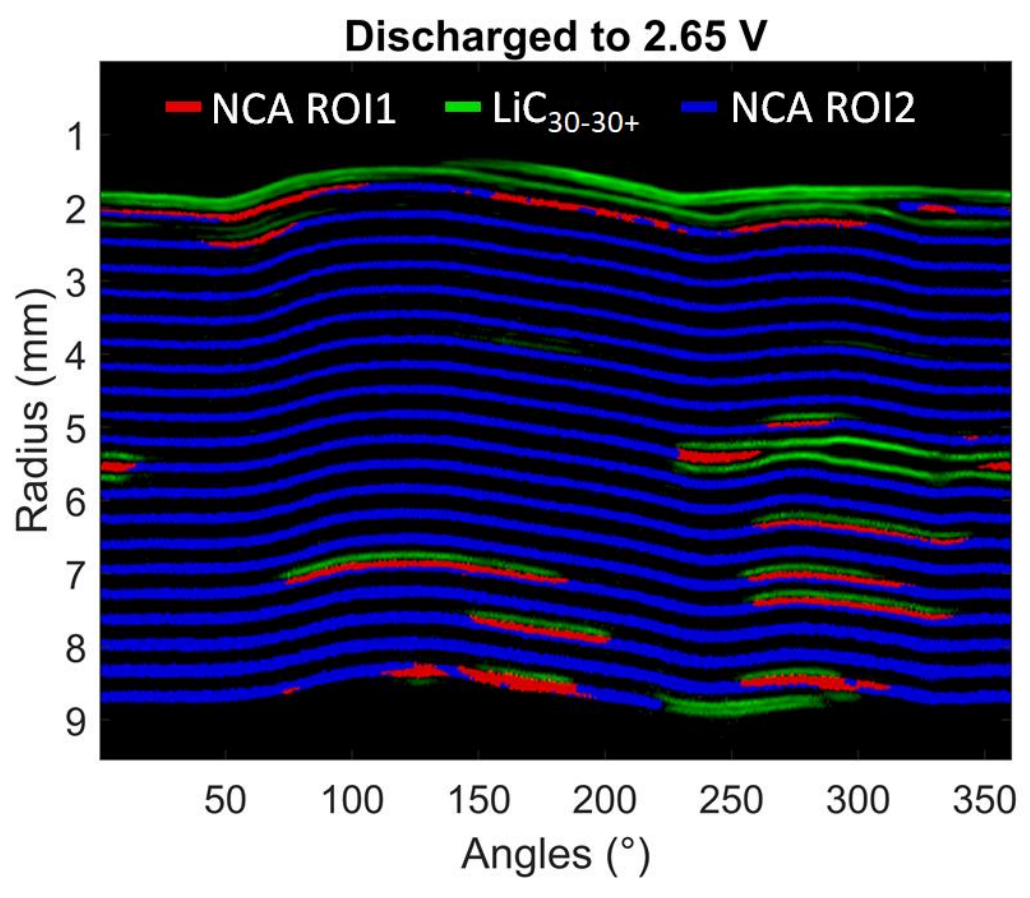


Figure 4. Distribution of lattice parameters a and $\mathrm{c}$ in the NCA phase for the battery cell charged to $4.2 \mathrm{~V}$ and discharged to $2.65 \mathrm{~V}$ during the first two cycles. Panel a: Spatial distribution obtained from the analysis of the XRD-CT data, Panel b: virtually unrolled battery electrode discharged to $2.65 \mathrm{~V}$ and presenting two regions of the NCA cathode material of different lattice parameters as well as the distribution of $\mathrm{LiC}_{30-30+}$ in the anode.

After the initial two cycles, four additional cycles were performed following the same experimental protocol. As expected, point XRD measurements and XRD line scans (Figure S8-S11) acquired during these four cycles did not indicate any deviations from the evolving solid-state chemistry reported above. In order to follow in situ the evolution of the $\mathrm{Li}_{x} \mathrm{C}$ crystalline phases and changes in NCA lattice parameters in the battery cross section, six XRD-CT datasets were collected while charging the battery (the 7th cycle) to various potentials $(3.7 \mathrm{~V}, 3.79 \mathrm{~V}, 3.85 \mathrm{~V}, 3.95 \mathrm{~V}$ and $4.2 \mathrm{~V})$, allowing for 10 minutes of cell relaxation under OCV after reaching the set potential. The evolution of the crystalline phases present in the anode is shown in Figure 5 (more information can be found in the Supporting Information Figure S12-S14). The spatial distribution of graphite and the two lithiated phases $\mathrm{LiC}_{30}$ and $\mathrm{LiC}_{30+}$ at the discharged cell $(2.65 \mathrm{~V})$ is in agreement with the previously reported results for the initial two cycles (Figure 3). After charging the cell to $3.7 \mathrm{~V}$, the graphite was seen to almost completely disappear while the amount of $\mathrm{LiC}_{30+}$ phase increased significantly, becoming uniformly distributed in the battery cross section. At $3.79 \mathrm{~V}, \mathrm{LiC}_{30}$ became the predominant phase while $\mathrm{LiC}_{30+}$ decreased significantly but remained in the anode in the regions previously identified to exhibit heterogeneities in lithium concentration (Al tab - separator). The formation of $\mathrm{LiC}_{18}$ in the anode was observed at $3.85 \mathrm{~V}$ (from XRD point measurements it was not possible to differentiate between $\mathrm{LiC}_{18}$ and $\mathrm{LiC}_{12}$ ) and it was uniformly distributed in the anode electrode. In addition, a small amount of $\mathrm{LiC}_{12}$ started to form at the electrode surface, following the same behaviour seen for the $\mathrm{LiC}_{6}$ (Figure 3). At $3.95 \mathrm{~V}$, four different $\mathrm{Li}_{x} \mathrm{C}$ phases were observed: $\mathrm{LiC}_{30+}, \mathrm{LiC}_{30}, \mathrm{LiC}_{18}$ and $\mathrm{LiC}_{12}$; from $3.95 \mathrm{~V}$ to $4.2 \mathrm{~V}$ the distribution of $\mathrm{LiC}_{30+}$ and $\mathrm{LiC}_{30}$ 
remained unchanged in the $\mathrm{Al}$ tab - separator region, while the $\mathrm{LiC}_{18}$ disappeared completely. At 3.95V, $\mathrm{LiC}_{12}$ became the predominant phase while a small amount of $\mathrm{LiC}_{6}$ formed at higher potentials above $3.95 \mathrm{~V}$ and primarily at the electrode surface. When comparing the distribution of the various $\mathrm{Li}_{x} \mathrm{C}$ phases during the in situ charging, three regions can be clearly distinguished in the anode exhibiting distinct properties: (1) uniform/homogenous lithiation (HL), (2) delayed lithiation (DL) and (3) inactive-to-lithiation (IN) (Figure S15). The region of uniform lithiation can be observed by the uniform distribution of $\mathrm{Li}_{x} \mathrm{C}$ phases progressing in the following order: graphite $\rightarrow \mathrm{LiC}_{30+} \rightarrow \mathrm{LiC}_{30} \rightarrow \mathrm{LiC}_{18} \rightarrow \mathrm{LiC}_{12}$ for the successive potentials during the in situ measurement. The region of delayed lithiation $(\mathrm{DL})$ can be located in the middle-region, however it is not present at 4.2 $\mathrm{V}$. The inactive region (IN) can be observed for the entire potential range (from $2.65 \mathrm{~V}$ to 4.2 $\mathrm{V}$ ) and can be identified from the distribution of $\mathrm{LiC}_{30-30+}$ in the charged state at $3.95 \mathrm{~V}$ and above. 


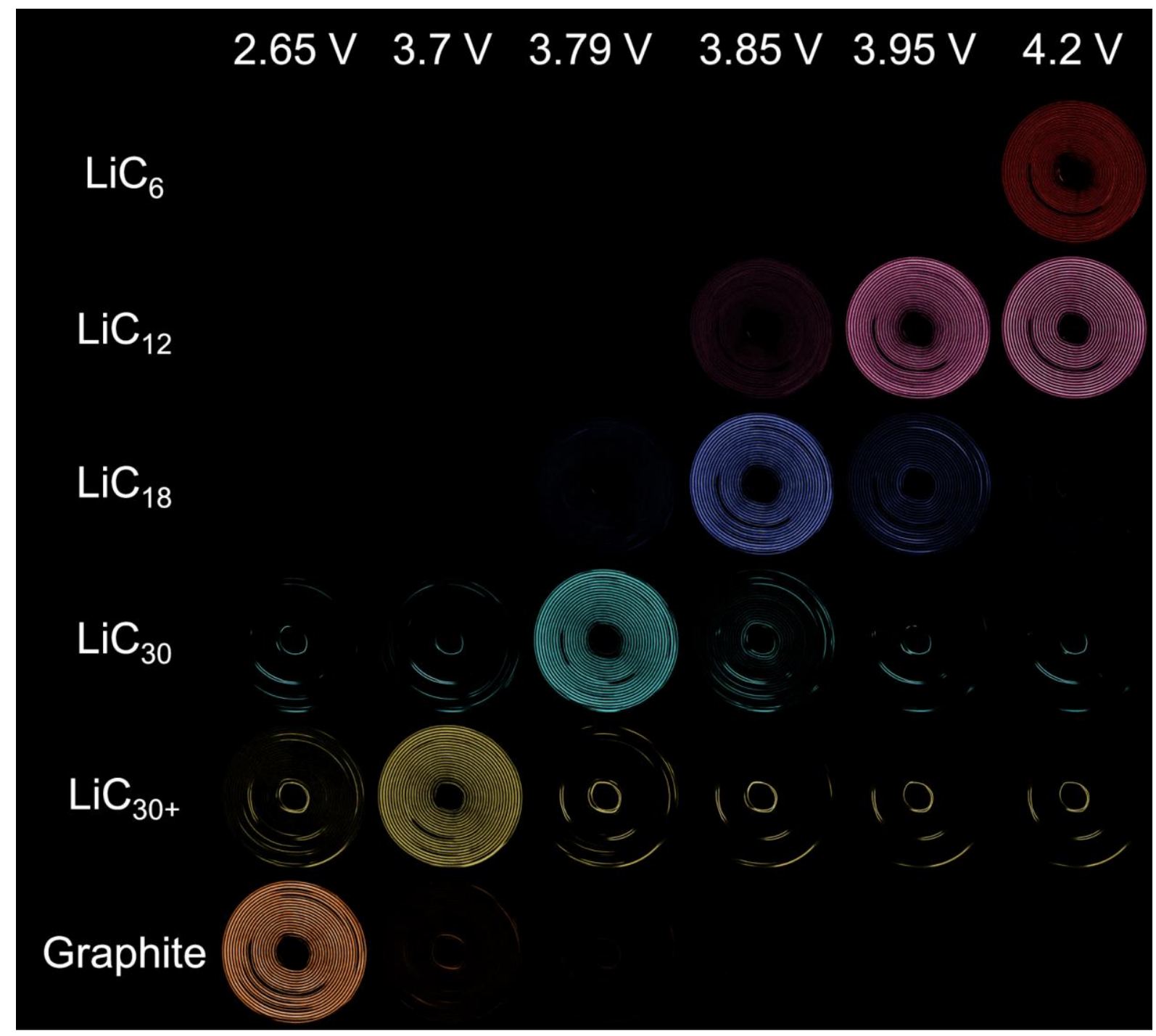

Figure 5. Spatial distribution of anode $\mathrm{Li}_{x} \mathrm{C}$ phases $\left(\mathrm{LiC}_{6}, \mathrm{LiC}_{12}, \mathrm{LiC}_{18}, \mathrm{LiC}_{30}, \mathrm{LiC}_{30+}\right.$ and graphite) as the cell is charged to various potentials, starting from $2.65 \mathrm{~V}$ and ending to $4.2 \mathrm{~V}$.

The spatial distribution of lattice parameters a and $\mathrm{c}$ of the NCA phase during charging to $4.2 \mathrm{~V}$ is presented in Figure 6. As previously reported for the discharged cell (Figure 4), two regions could be observed exhibiting different lattice parameters: (1) cathode directly connected to the Al tab and few layers in the middle-region (ROI1) and (2) the rest of the electrode layers (ROI2), the latter exhibiting higher lattice parameter a and lower lattice parameter c suggesting more lithium in its structure (diffraction patterns from these regions can be found in the Supporting Information 
Figure S16-S18). From 2.65 V to $3.7 \mathrm{~V}$ no significant changes in the lattice parameters a and $\mathrm{c}$ were observed. At $3.79 \mathrm{~V}$ and $3.85 \mathrm{~V}$ the $\mathrm{ROI} 2$ region experienced significant changes in the lattice parameters of the hexagonal NCA phase, reaching values close to the ROI1 region (i.e. decrease in lattice parameter a and increase in lattice parameter c was observed, which is typical for the layered materials during charging i.e. while lithium is being removed from their structure). From 3.95 V, the two regions related to the lattice parameters distribution changed; the ROI1 region now contained only the cathode material directly connected to the Al tab, while the ROI2 region consisted of the rest of the cathode. This change is coincidental with the disappearance of the $\mathrm{DL}$ region from the anode, as discussed in the section above, which is in close proximity to the region of the cathode that has experienced these changes (middle-region). In addition, in the ROI2 the lattice parameter a continued to decrease (with the corresponding increase in lattice parameter c) while in the ROI1 region an increase in lattice parameter a and a decrease in lattice parameter c could be observed. 

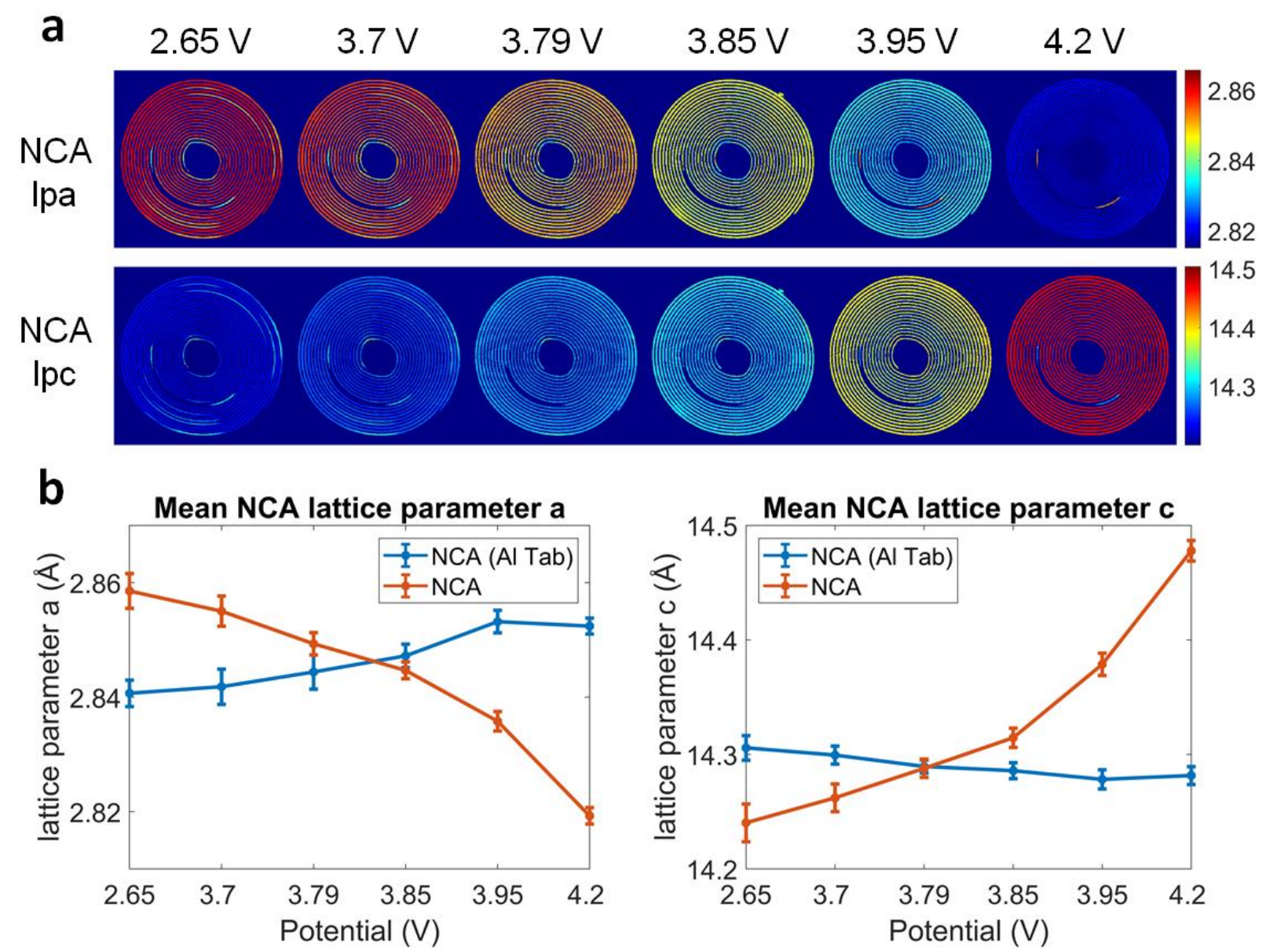

Figure 6. Evolution of lattice parameters a and $c$ of the NCA phase for the battery cell charged to various potentials from $2.65 \mathrm{~V}$ to $4.2 \mathrm{~V}$. Panel a: Spatial distribution of NCA lattice parameters and Panel b: Mean lattice parameter values from two distinct NCA regions: in close proximity to the Al tab and the rest of the electrode.

The in situ XRD-CT measurements performed while charging the battery to $4.2 \mathrm{~V}$ revealed heterogeneities in lithium distribution in both the cathode and the anode. As discussed in the previous section, there are two distinct regions (ROI1 and $\mathrm{ROI}$ ) in the cathode exhibiting different behaviour during de-lithiation. The ROI1 region is related to cathode material directly connected to the Al tab. In this region, the lattice parameters are experiencing only a minor change during the charging protocol. However it should be noted that the cathode is exhibiting the opposite 
behaviour to what is expected during charging of NCA. Specifically, an increase in lattice parameter a and decrease in lattice parameter $\mathrm{c}$ is observed as opposed to the typical behaviour which is decrease in lattice parameter a and increase in lattice parameter c. Heterogeneities related to the thickness of the cathode coating near the Al current collector have been previously suggested to lead to local variations in charge- and discharge current densities which can be associated with battery degradation ${ }^{53-57}$. The rest of the cathode material (ROI2) exhibits typical changes in the lattice parameters of hexagonal structure suggesting that the close proximity of the aluminum tab directly affects the lithium distribution in the cathode material. It is important to note that no other phases (hexagonal or monoclinic) were observed during the experiment.

Regarding the lithium distribution in the anode, this can be directly visualised through the evolution (formation, growth and disappearance) of the various $\mathrm{Li}_{x} \mathrm{C}$ phases; three regions exhibiting different properties were identified and defined previously as $\mathrm{HL}, \mathrm{DL}$ and IN respectively. It is expected that both the $\mathrm{DL}$ region and the $\mathrm{IN}$ region will have a negative impact on the cell performance. The $\mathrm{DL}$ region is present primarily at voltages in the range between $2.65 \mathrm{~V}$ and 3.95 $\mathrm{V}$ (no presence of this region was observed at $4.2 \mathrm{~V}$ ) and its presence was seen to affect the distribution of lithium in the closely located cathode material; at the discharge state it was shown that lithium stays in this region in the form of $\mathrm{LiC}_{30-30+}$, preventing the complete lithiation of cathode material. However, at higher voltages (above $3.95 \mathrm{~V}$ ) this region does not seem to contribute to the formation of lithium heterogeneities. Regarding the IN region, it was found to be present from the beginning of the cell operation and was located in proximity to the Al tab - separator region. Throughout the seven cycles performed in this work, no changes were observed related to the distribution of this region. The lithium incorporated in the anode as a $\mathrm{LiC}_{30-30+}$ phase was most likely formed during the initial cycle performed by the manufacturer and is a result of the cell design; the influence of the Al current collector, stainless steel casing and separator at the core of the jelly roll (Fe-Al-Sep) region was shown previously to affect the lithium distribution in a 
commercial AAA Li-ion battery ${ }^{42}$. Our investigation shows that significant heterogeneities in the lithium distribution can be expected to form primarily in the anode during charging and discharging. These heterogeneities can contribute both to the capacity loss at the anode side as well as affect the distribution of lithium in the cathode.

\section{Conclusions}

We employed the XRD-CT technique to study, for the first time, the evolving solid-state chemistry of a commercial cylindrical 18650 Li-ion battery under operating conditions. More specifically, we applied a high resolution diffraction imaging technique to investigate both the cathode and anode as well as the multiple heterogeneities in the lithium distribution within each electrode and in the cell cross section. The very large (ca. 4,000,000) and rich diffraction (13 phases identified) data obtained through diffraction imaging were analysed with the state-of-the-art Parameter Quantification neural network (PQ-Net) which allowed for semi-automated and ultra-fast data analysis resolving the evolution of the complex chemistry of this multi-component system. The lithium distribution at the anode side was observed indirectly through the distribution of various crystalline $\mathrm{Li}_{x} \mathrm{C}$ phases while the lithium distribution at the cathode side was observed from the distribution of the lattice parameters of the hexagonal $\mathrm{LiNi}_{0.8} \mathrm{Co}_{0.15} \mathrm{Al}_{0.5} \mathrm{O}_{2}$ (NCA) phase. Our investigation revealed the presence of three different regions in the anode affecting its ability to interact with lithium. The inactive-to-lithiation region is primarily a result of the cell geometry, as the aluminum tab and separator were identified in its close proximity. While the presence of a delayed-lithiation region at lower voltages $(<4.0 \mathrm{~V})$ cannot be explained by the cell geometry, its occurrence was seen to negatively affect the distribution of lithium at the cathode side in the layers adjacent to it, at both the charged and discharged states. It should be emphasized that it was the combination of in situ measurements and XRD-CT that allowed us to identify this key finding; it is possible that degradation of battery (capacity fade and/or cell failure) will initialise in this region. 
This could allow for early-on identification of potential regions where cell degradation will develop before there is an indication through measurements of macroscopic properties (e.g. capacity fade from cycling stability measurements). Further to this, the presence of the Al tab affected the distribution of lithium in the cathode material being in direct contact with it, preventing lithium insertion during discharging and trapping the lithium during charging. This research highlights the impact of cell geometry on the initial capacity loss which is indicated to be primarily driven by the heterogeneities at the anode side. Finally, it should be noted that XRD-CT has the potential to be applied for a wide variety of different experiments related to battery degradation mechanisms, such as in situ/ operando abusing testing to capture internal temperature gradients through the crystallographic behaviour of the anode/cathode as well as ex situ measurements to investigate active layer delamination and local corrosion of spent samples.

\section{Methods \& Materials}

\section{XRD-CT measurements}

XRD-CT measurements were performed at beamline ID31 of the ESRF using a $95 \mathrm{keV}(\lambda=$ $0.130509 \AA$ ) monochromatic X-ray beam focused to have a spot size of ca. $25 \times 4 \mu \mathrm{m}$ (Horizontal $x$ Vertical). 2D powder diffraction patterns were acquired using the Pilatus3 X CdTe $2 \mathrm{M}$ hybrid photon counting area detector. The battery was mounted onto a goniometer which was placed on a rotation stage (Figure S19) itself mounted on top of a translation stage. The sample-to-detector distance was approximately $1 \mathrm{~m}$. The XRD-CT scans were measured by performing a series of zigzag line scans using the interlaced approach ${ }^{58}$. An exposure time of $10 \mathrm{~ms}$ and an angular range of $0-180^{\circ}$ with 400 projections in total were used for each XRD-CT dataset. Four interlaced XRD-CT subsets were collected, each with 100 projections covering an angular range of $0-180^{\circ}$ using the same angular step size and a different starting angle. The fast axis was the translation 
axis (fly scanning) and slow axis was the rotation axis. For each interlaced zigzag XRD-CT scan a translation step size of 30 microns was applied ${ }^{58}$; in total 637 translation steps were made per line scans corresponding to a covered area of $19.11 \times 19.11 \mathrm{~mm}^{2}$. The detector calibration was performed using a $\mathrm{CeO}_{2}$ standard (Figure S20). Every 2D diffraction image was calibrated and azimuthally integrated to a 1D powder diffraction pattern with a $20 \%$ trimmed mean filter using the pyFAI software package and in-house developed scripts ${ }^{59-61}$. The integrated diffraction patterns were reshaped into sinograms and centered; the air scatter signal was subtracted from the data. The XRD-CT images were initially reconstructed using the filtered back projection algorithm in MATLAB. The voxel size in the reconstructed images corresponded to $30 \times 30 \times 4$ $\mu \mathrm{m}^{3}$. To further illustrate the chemical gradients present in the XRD-CT data, images-of-interest were converted from cartesian to polar coordinates using inhouse MATLAB developed scripts. This approach enables the virtual unrolling of the battery cell jelly-roll providing means to capture gradients as a function of the battery radius ${ }^{62}$.

Self-absorption correction, tomographic image denoising and diffraction data analysis

The XRD-CT data were first analysed using the conventional approach; the images were reconstructed using the filtered back projection algorithm and then full profile analysis of the local diffraction patterns was performed using the Rietveld method. This type of analysis can lead to accurate scale factor and weight fraction maps for the various crystalline components in the sample despite the parallax artefacts present in the data. The weight fraction maps obtained from the Rietveld analysis were used to simulate an absorption map using the X-ray mass attenuation coefficient from NIST ${ }^{63}$. This $\mu$ attenuation map was then used to correct the XRD-CT sinogram data for self-absorption using the zero-order approximation (Figures S21-23) ${ }^{64}$. This process was followed for all acquired XRD-CT datasets. The self-absorption corrected XRD-CT sinograms were used to reconstruct high quality XRD-CT images (Figures S24-25) using a modified version of the Noise2Inverse denoising self-supervised convolutional neural network ${ }^{65,66}$. The denoised 
XRD-CT data were then passed to PQNet models for analysis of the local diffraction patterns. It should be noted that the XRD-CT data contain parallax artefacts due to the large sample size which can introduce errors related to peak position and peak shape. In this work it was not possible to use the DLSR algorithm due to computational limitations i.e. it is not currently computationally feasible to process $637 \times 637$ images and rebinning the data to lower resolution leads to unstable results due to the thickness of the electrode layer being very thin. To suppress the parallax artefact, the $Q$ range used for the data analysis in this work was restricted to a region of $0.5-3.85 \AA^{-1}$. The differences between the two distinct regions in the NCA lattice parameters maps presented in this work are not parallax related as clearly indicated by the error bars in Figure 6 (i.e. the errors correspond to the standard deviation). Quantitative Rietveld refinement was performed on reconstructed diffraction patterns with the TOPAS v7 software ${ }^{67}$ (Figures S3-4). The PQNet was used to analyse the spatially-resolved diffraction patterns present in all XRD-CT datasets presented in this work. Details regarding the network architectures and parameters used are provided in the Supporting Information (Figures S26-28). A pseudo-voigt peak shape function calculated after the analysis of the $\mathrm{CeO}_{2}$ calibrant pattern was used for diffraction data (Figure S20). A single pseudo-voigt peak was used to model the peak generated by the semi-crystalline separator component.

Electrochemical testing and sample description

A Samsung 35E 3500 mAh 3.7 V battery (18650) was investigated in this work. The dimensions of the cells are specified as $18.3 \times 65 \mathrm{~mm}$ (diameter $\times$ height). The composition of the cathode electrode was determined with ex situ X-ray fluorescence spectroscopy measurements (Figures S29 and Table S6). The elemental analysis revealed that the cathode consists of $\mathrm{LiNi}_{0.8} \mathrm{Al}_{0.15} \mathrm{Co}_{0.05} \mathrm{O}_{2}$ (NCA). Electrochemical cycling was carried out using a Biologic SP240 Potentiostat equipped with a current booster to 4 A (BioLogic Sciences Instruments, France). The 
as-received battery was first discharged to $2.65 \mathrm{~V}$ with CC-CV protocol applying $700 \mathrm{~mA}$ current. The cell was cycled within the voltage limits specified by the manufacturer, $2.65 \mathrm{~V}$ to $4.2 \mathrm{~V}$ respectively. The cycling protocol included applying a constant current of $3.5 \mathrm{~A}$ (1 C rate with respect to the nominal capacity stated by the manufacturer) for the charge and $-3.5 \mathrm{~A}$ for the discharge. After reaching the set potential (4.2 V for charged state and $2.65 \mathrm{~V}$ to discharged state) the applied current was removed and the cell was allowed to relax for 10 minutes (OCV). Then the XRD-CT measurements were performed, each scan lasting approximately $1 \mathrm{hr}$. The charge capacity was estimated to be ca. $65 \%$ of the nominal capacity provided by the manufacturer (2283 / 3500mAh $=65 \%)$.

\section{Author contributions}

D.M., A.V. and T.A. conceived the experiment. The XRD-CT measurements were performed by D.M., A.V., M.M., I.M. and J.Dr.. The laboratory x-ray fluorescence spectroscopy measurements were performed by T.A. M.M., I.M. and J.Dr. were responsible for ID31 instrumentation and setup at the ESRF. The MATLAB/TOPAS scripts were developed and optimised by A.V. and D.M. H.D. and A.V. developed and applied the PQNet models used in this work. H.D. and A.V. implemented and applied the modified Noise2Inverse neural network used in this work. The XRD-CT data were analysed by A.V. and D.M. with contributions from H.D., J.D., S.D.M.J and A.M.B.. D.M., A.V. and T.A. are responsible for writing the manuscript with contributions and feedback given by all contributors. A.V. directed the research.

\section{Acknowledgements}

We acknowledge ESRF for provision of beamtime. The authors would like to thank Allard Hendriksen for advising on the Noise2 Inverse implementation for tomographic data denoising. 
AMB acknowledges funding from EPSRC, grant awards EP/S016481/1 \& EP/R026939/1. JAD and TA acknowledge funding from the JUICED energy Hub (EP/R023662/1).

\section{Data Availability}

All data are available from the corresponding authors on reasonable request.

\section{References}

1. Zubi, G., Dufo-López, R., Carvalho, M. \& Pasaoglu, G. The lithium-ion battery: State of the art and future perspectives. Renew. Sustain. Energy Rev. 89, 292-308 (2018).

2. Myung, S.-T. et al. Nickel-Rich Layered Cathode Materials for Automotive Lithium-Ion Batteries: Achievements and Perspectives. ACS Energy Lett. 2, 196-223 (2017).

3. Kim, T., Song, W., Son, D.-Y., Ono, L. K. \& Qi, Y. Lithium-ion batteries: outlook on present, future, and hybridized technologies. J. Mater. Chem. A 7, 2942-2964 (2019).

4. Cutting cobalt. Nat. Energy 5, 825-825 (2020).

5. Masias, A., Marcicki, J. \& Paxton, W. A. Opportunities and Challenges of Lithium lon Batteries in Automotive Applications. ACS Energy Lett. 6, 621-630 (2021).

6. Miao, Y., Hynan, P., von Jouanne, A. \& Yokochi, A. Current Li-Ion Battery Technologies in Electric Vehicles and Opportunities for Advancements. Energies 12, 1074 (2019).

7. Park, K.-J. et al. Degradation Mechanism of Ni-Enriched NCA Cathode for Lithium Batteries: Are Microcracks Really Critical? ACS Energy Lett. 4, 1394-1400 (2019).

8. Pender, J. P. et al. Electrode Degradation in Lithium-Ion Batteries. ACS Nano 14, 12431295 (2020).

9. Kim, J.-H., Ryu, H.-H., Kim, S. J., Yoon, C. S. \& Sun, Y.-K. Degradation Mechanism of Highly Ni-Rich Li[NixCoyMn1-x-y]O2 Cathodes with x > 0.9. ACS Appl. Mater. Interfaces 
11, 30936-30942 (2019).

10. Li, T. et al. Degradation Mechanisms and Mitigation Strategies of Nickel-Rich NMC-Based Lithium-Ion Batteries. Electrochem. Energy Rev. 3, 43-80 (2020).

11. Palacín, M. R. Understanding ageing in Li-ion batteries: a chemical issue. Chem. Soc. Rev. 47, 4924-4933 (2018).

12. Zhang, G. et al. In Situ Measurement of Radial Temperature Distributions in Cylindrical LiIon Cells. J. Electrochem. Soc. 161, A1499 (2014).

13. Drake, S. J. et al. Measurement of anisotropic thermophysical properties of cylindrical Li-ion cells. J. Power Sources 252, 298-304 (2014).

14. Kim, H.-K., Choi, J. H. \& Lee, K.-J. A Numerical Study of the Effects of Cell Formats on the Cycle Life of Lithium Ion Batteries. J. Electrochem. Soc. 166, A1769 (2019).

15. Rheinfeld, A. et al. Impact of Cell Size and Format on External Short Circuit Behavior of Lithium-Ion Cells at Varying Cooling Conditions: Modeling and Simulation. J. Electrochem. Soc. 167, 013511 (2019).

16. Zhang, Y., Yang, Z. \& Tian, C. Probing and quantifying cathode charge heterogeneity in Li ion batteries. J. Mater. Chem. A 7, 23628-23661 (2019).

17. Sheng, Y. et al. Effect of Calendering on Electrode Wettability in Lithium-lon Batteries. Front. Energy Res. 0, (2014).

18. Heenan, T. M. M. et al. Identifying the Origins of Microstructural Defects Such as Cracking within Ni-Rich NMC811 Cathode Particles for Lithium-Ion Batteries. Adv. Energy Mater. 10, 2002655 (2020).

19. Günther, T. et al. Classification of Calendering-Induced Electrode Defects and Their Influence on Subsequent Processes of Lithium-Ion Battery Production. Energy Technol. 8, 1900026 (2020).

20. Tan, C. C. et al. Ageing analysis and asymmetric stress considerations for small format cylindrical cells for wearable electronic devices. J. Power Sources 472, 228626 (2020). 
21. Klett, M. et al. Non-uniform aging of cycled commercial LiFePO4//graphite cylindrical cells revealed by post-mortem analysis. J. Power Sources 257, 126-137 (2014).

22. Barkholtz, H. M. et al. Multi-scale thermal stability study of commercial lithium-ion batteries as a function of cathode chemistry and state-of-charge. J. Power Sources 435, 226777 (2019).

23. Sørensen, D. R. et al. Fatigue in High-Energy Commercial Li Batteries while Cycling at Standard Conditions: An In Situ Neutron Powder Diffraction Study. ACS Appl. Energy Mater. 3, 6611-6622 (2020).

24. Bobrikov, I. A. et al. In-situ time-of-flight neutron diffraction study of the structure evolution of electrode materials in a commercial battery with LiNi0.8Co0.15Al0.05O2 cathode. J. Power Sources 372, 74-81 (2017).

25. Gelb, J., Finegan, D. P., Brett, D. J. L. \& Shearing, P. R. Multi-scale 3D investigations of a commercial 18650 Li-ion battery with correlative electron- and X-ray microscopy. J. Power Sources 357, 77-86 (2017).

26. Lin, F. et al. Synchrotron X-ray Analytical Techniques for Studying Materials Electrochemistry in Rechargeable Batteries. Chem. Rev. 117, 13123-13186 (2017).

27. Dolotko, O., Senyshyn, A., Mühlbauer, M. J., Nikolowski, K. \& Ehrenberg, H. Understanding structural changes in NMC Li-ion cells by in situ neutron diffraction. J. Power Sources 255 , 197-203 (2014).

28. Taminato, S. et al. Real-time observations of lithium battery reactions-operando neutron diffraction analysis during practical operation. Sci. Rep. 6, 28843 (2016).

29. Deng, Z. et al. Recent Progress on Advanced Imaging Techniques for Lithium-lon Batteries. Adv. Energy Mater. 11, 2000806 (2021).

30. Finegan, D. P. et al. In-operando high-speed tomography of lithium-ion batteries during thermal runaway. Nat. Commun. 6, 6924 (2015).

31. Ziesche, R. F. et al. 4D imaging of lithium-batteries using correlative neutron and X-ray 
tomography with a virtual unrolling technique. Nat. Commun. 11, 777 (2020).

32. Waldmann, T., Scurtu, R.-G., Richter, K. \& Wohlfahrt-Mehrens, M. 18650 vs. 21700 Li-ion cells - A direct comparison of electrochemical, thermal, and geometrical properties. J. Power Sources 472, 228614 (2020).

33. Wang, X.-L. et al. Visualizing the chemistry and structure dynamics in lithium-ion batteries by in-situ neutron diffraction. Sci. Rep. 2, 747 (2012).

34. Sheptyakov, D. et al. Stroboscopic neutron diffraction applied to fast time-resolved operando studies on Li-ion batteries (d-LiNi0.5Mn1.5O4vs. graphite). J. Mater. Chem. A 8, 1288-1297 (2020).

35. Kimura, Y. et al. Influence of Active Material Loading on Electrochemical Reactions in Composite Solid-State Battery Electrodes Revealed by Operando 3D CT-XANES Imaging. ACS Appl. Energy Mater. 3, 7782-7793 (2020).

36. Sottmann, J. et al. Chemical Structures of Specific Sodium Ion Battery Components Determined by Operando Pair Distribution Function and X-ray Diffraction Computed Tomography. Angew. Chem. Int. Ed. 56, 11385-11389 (2017).

37. Petz, D. et al. Lithium heterogeneities in cylinder-type Li-ion batteries - fatigue induced by cycling. J. Power Sources 448, 227466 (2020).

38. Petz, D. et al. Lithium distribution and transfer in high-power 18650-type Li-ion cells at multiple length scales. Energy Storage Mater. 41, 546-553 (2021).

39. Mühlbauer, M. J. et al. Inhomogeneous distribution of lithium and electrolyte in aged Li-ion cylindrical cells. J. Power Sources 475, 228690 (2020).

40. Senyshyn, A., Mühlbauer, M. J., Dolotko, O., Hofmann, M. \& Ehrenberg, H. Homogeneity of lithium distribution in cylinder-type Li-ion batteries. Sci. Rep. 5, 18380 (2015).

41. Dong, H. et al. A deep convolutional neural network for real-time full profile analysis of big powder diffraction data. Npj Comput. Mater. 7, 74 (2021).

42. Vamvakeros, A. et al. Cycling rate-induced spatially-resolved heterogeneities in commercial 
cylindrical Li-ion batteries. Small Methods (2021) doi:10.1002/smtd.202100512.

43. Vadlamani, B., An, K., Jagannathan, M. \& Chandran, K. S. R. An In-Situ Electrochemical Cell for Neutron Diffraction Studies of Phase Transitions in Small Volume Electrodes of LiIon Batteries. J. Electrochem. Soc. 161, A1731 (2014).

44. Didier, C., Pang, W. K., Guo, Z., Schmid, S. \& Peterson, V. K. Phase Evolution and Intermittent Disorder in Electrochemically Lithiated Graphite Determined Using in Operando Neutron Diffraction. Chem. Mater. 32, 2518-2531 (2020).

45. Missyul, A., Bolshakov, I. \& Shpanchenko, R. XRD study of phase transformations in lithiated graphite anodes by Rietveld method. Powder Diffr. 32, S56-S62 (2017).

46. Boulet-Roblin, L. et al. Crystal structure evolution via operando neutron diffraction during long-term cycling of a customized $5 \mathrm{~V}$ full Li-ion cylindrical cell LiNi0.5Mn1.5O4vs. graphite. J. Mater. Chem. A 5, 25574-25582 (2017).

47. Zinth, V. et al. Inhomogeneity and relaxation phenomena in the graphite anode of a lithiumion battery probed by in situ neutron diffraction. J. Power Sources 361, 54-60 (2017).

48. Yao, X.-Y. \& Pecht, M. G. Tab Design and Failures in Cylindrical Li-ion Batteries. IEEE Access 7, 24082-24095 (2019).

49. Finegan, D. P. et al. Spatial dynamics of lithiation and lithium plating during high-rate operation of graphite electrodes. Energy Environ. Sci. 13, 2570-2584 (2020).

50. Finegan, D. P. et al. Spatially Resolving Lithiation in Silicon-Graphite Composite Electrodes via in Situ High-Energy X-ray Diffraction Computed Tomography. Nano Lett. 19, 3811-3820 (2019).

51. Hwang, S. \& Stach, E. A. Usingin situand operando methods to characterize phase changes in charged lithium nickel cobalt aluminum oxide cathode materials. J. Phys. Appl. Phys. 53, $113002(2020)$.

52. Kleiner, K. et al. Fatigue of LiNi0.8Co0.15Al0.05O2 in commercial Li ion batteries. J. Power Sources 273, 70-82 (2015). 
53. Pfrang, A. et al. Long-term cycling induced jelly roll deformation in commercial 18650 cells. J. Power Sources 392, 168-175 (2018).

54. Aurbach, D. et al. An analysis of rechargeable lithium-ion batteries after prolonged cycling. Electrochimica Acta 47, 1899-1911 (2002).

55. Wohlfahrt-Mehrens, M., Vogler, C. \& Garche, J. Aging mechanisms of lithium cathode materials. Eighth Ulm. Electrochem. Tage 127, 58-64 (2004).

56. Ecker, M. et al. Calendar and cycle life study of Li(NiMnCo)O2-based 18650 lithium-ion batteries. J. Power Sources 248, 839-851 (2014).

57. Barré, A. et al. A review on lithium-ion battery ageing mechanisms and estimations for automotive applications. J. Power Sources 241, 680-689 (2013).

58. Vamvakeros, A. et al. Interlaced X-ray diffraction computed tomography. J. Appl. Crystallogr. 49, (2016).

59. Vamvakeros, A. et al. Removing multiple outliers and single-crystal artefacts from X-ray diffraction computed tomography data. J. Appl. Crystallogr. 48, 1943-1955 (2015).

60. Ashiotis, G. et al. The fast azimuthal integration Python library: PyFAl. J. Appl. Crystallogr. 48, 510-519 (2015).

61. Vamvakeros, A. nDTomo Software Suite. (2018).

62. Kok, M. D. R. et al. Virtual unrolling of spirally-wound lithium-ion cells for correlative degradation studies and predictive fault detection. Sustain. Energy Fuels 3, 2972-2976 (2019).

63. Linstrom, P. J. \& Mallard, W. G. NIST Chemistry WebBook. NIST Stand. Ref. Database Number 69 doi:https://doi.org/10.18434/T4D303.

64. Vamvakeros, A. et al. Real-time tomographic diffraction imaging of catalytic membrane reactors for the oxidative coupling of methane. Catal. Today 364, 242-255 (2021).

65. A. A. Hendriksen, D. M. Pelt, \& K. J. Batenburg. Noise2Inverse: Self-Supervised Deep Convolutional Denoising for Tomography. IEEE Trans. Comput. Imaging 6, 1320-1335 
(2020).

66. Hendriksen, A. A. et al. Deep denoising for multi-dimensional synchrotron X-ray tomography without high-quality reference data. Sci. Rep. 11, 11895 (2021).

67. Coelho, A. TOPAS and TOPAS-Academic: an optimization program integrating computer algebra and crystallographic objects written in C++. J. Appl. Crystallogr. 51, 210-218 (2018). 